\title{
3. Funktionale und subjektive Gesundheit bei Frauen und Männern im Verlauf der zweiten Lebenshälfte
}

\author{
Svenja M. Spuling, Anja Cengia und Markus Wettstein
}

\section{Kernaussagen}

Mit steigendem Alter berichten Frauen und Männer eine zunehmend eingeschränkte funktionale Gesundheit, und sie bewerten ihre Gesundheit weniger positiv: Die funktionale Gesundheit, das heißt die selbstständige Mobilität und die allgemeine Alltagskompetenz, nimmt mit zunehmendem Alter ab. Auch die individuelle subjektive Bewertung der eigenen Gesundheit verschlechtert sich mit zunehmendem Alter, sie nimmt aber über den Alternsverlauf von 40 bis 90 Jahren insgesamt weniger stark ab als die funktionale Gesundheit.

Frauen berichten zwar eine stärker eingeschränkte funktionale Gesundheit als Männer - und das im Alternsverlauf mit zunehmenden Maße -, aber subjektiv bewerten Frauen und Männer ihre Gesundheit ähnlich über die gesamte zweite Lebenshälfte: Frauen haben bereits im Alter von 40 Jahren eine schlechtere funktionale Gesundheit als Männer, und dieser Geschlechterunterschied nimmt mit steigendem Alter zu. Dagegen unterscheiden sich Frauen und Männer weder im Alter von 40 Jahren noch im Alternsverlauf hinsichtlich ihrer subjektiven Gesundheit. Frauen und Männer bewerten ihre Gesundheit demnach subjektiv ähnlich, obwohl sie sich in ihrer funktionalen Gesundheit unterscheiden.

Später Geborene kommen voraussichtlich mit einer besseren funktionalen Gesundheit ins höhere Alter als früher Geborene: In der ersten Hälfte des fünften Lebensjahrzehnts haben früher geborene Kohorten eine durchschnittlich bessere funktionale Gesundheit als später geborene Kohorten. Dies gilt für Frauen und Männer gleichermaßen. Im höheren Alter werden jedoch Frauen und Männer später geborener Kohorten voraussichtlich eine bessere funktionale Gesundheit und eine geringere Abnahme der funktionalen Gesundheit aufweisen als die Frauen und Männer früher geborener Kohorten. Dagegen unterscheidet sich bei der subjektiven Gesundheit weder das mittlere Ausgangsniveau mit Anfang 40 noch der Alternsverlauf zwischen den untersuchten Geburtskohorten, dies gilt für Frauen und Männer. 


\subsection{Einleitung}

Gesundheit hat für alle Menschen einen hohen Stellenwert, doch oft wird ihr erst in der zweiten Lebenshälfte Aufmerksamkeit zuteil (Böhm, TeschRömer, \& Ziese 2009). Der eigene Gesundheitszustand beeinflusst zum einen maßgeblich viele persönliche Lebensbereiche: Gesunde Menschen leben meist selbstständiger als Menschen, die gesundheitlich eingeschränkt sind. Für Gesunde ist das Risiko, hilfs- und pflegebedürftig zu werden, geringer, ihr allgemeines Wohlbefinden ist höher und sie können ihre Freizeit autonom gestalten und soziale Kontakte pflegen (Schöllgen, Morack, Infurna, Ram, \& Gerstorf 2016). Zum anderen hat die Gesundheit eine hohe soziale Relevanz, weil sie sich auch darauf auswirkt, ob Menschen an der Gesellschaft teilhaben, sich freiwillig engagieren oder für andere sorgen können.

Lange Zeit wurde Gesundheit mit der Abwesenheit körperlicher Erkrankungen gleichgesetzt. Zur Definition dessen, was unter Gesundheit zu verstehen ist, wurden ausschließlich medizinische Gesundheitsmodelle herangezogen, denen eine eindimensionale Vorstellung von "gesund“ versus „krank“ zugrunde liegt. Um den Gesundheitszustand von Menschen in seiner ganzen Breite zu erfassen, ist jedoch ein mehrdimensionales Konzept von Gesundheit erforderlich, das neben der körperlichen Gesundheit etwa auch die körperliche Funktionsfähigkeit, die subjektive Gesundheitsbewertung sowie die psychische Gesundheit berücksichtigt (vgl. World Health Organization 2002). Im vorliegenden Kapitel werden die beiden Dimensionen der funktionalen Gesundheit und der subjektiven Gesundheit eingehender beleuchtet, Informationen zur psychischen Gesundheit bietet das Kapitel 4 Lebenszufriedenheit und depressive Symptome in diesem Band.

Gesundheitszustände können sich zum einen über den Lebensverlauf verändern und zum anderen zwischen den Geschlechtern unterscheiden. Zudem differieren womöglich die Veränderungen in der Gesundheit von Frauen und Männern mit zunehmendem Alter. Um individuelle gesund- heitliche Veränderungen mit zunehmendem Alter umfassend abbilden zu können, ist ein langer Beobachtungszeitraum mit wiederholten Befragungen wichtig.

\section{Funktionale und subjektive Gesundheit im Alter}

Beim Konzept der funktionalen Gesundheit wird die körperliche Funktionsfähigkeit im Alltag betrachtet. Menschen sind funktional gesund, wenn sie in ihrer Alltagsmobilität, in ihrer selbstständigen Lebensführung beziehungsweise der allgemeinen Alltagskompetenz nicht eingeschränkt sind. Einschränkungen der funktionalen Gesundheit liegen vor, wenn Menschen Alltagsaufgaben, wie zum Beispiel Einkaufengehen, Treppensteigen, Putzen, Waschen oder Nahrungszubereitung und -aufnahme, nicht ohne fremde Hilfe bewältigen können (Baltes, Maas, Wilms, \& Borchelt 2010). Die körperliche Gesundheit (im Sinne der Anzahl an chronischen Erkrankungen) ist dabei auch ein Einflussfaktor - je schlechter die körperliche Gesundheit ist, desto wahrscheinlicher ist es, dass jemand im Alltag funktional eingeschränkt ist. Dabei bleibt aber zu beachten, dass allein das Vorhandensein einer oder mehrerer Erkrankungen nicht unbedingt zu einer funktionalen Einschränkung führen muss - viele Personen meistern auch mit mehreren Erkrankungen ihren Alltag ohne jegliche Einschränkungen.

Mit zunehmendem Alter berichten deutlich mehr Menschen Einschränkungen bei alltäglichen Aktivitäten (Wolff, Nowossadeck, \& Spuling 2017). Eine mögliche Erklärung hierfür ist das häufigere Auftreten von Multimorbidität - also das Vorliegen mehrerer Erkrankungen gleichzeitig - mit zunehmendem Alter, welche das Risiko für das Auftreten von funktionalen Einschränkungen erhöht. Durch altersphysiologische Prozesse, also biologische Veränderungen des alternden Körpers, erhöht sich mit zunehmendem Alter die Wahrscheinlich- 
keit, an chronischen Erkrankungen zu leiden sowie von Multimorbidität betroffen zu sein (Barnett, Mercer, Norbury, Watt, Wyke, \& Guthrie 2012). Diese altersphysiologischen Prozesse umfassen typischerweise unter anderem die Abnahme von Knochendichte, Muskelkraft, Arterienelastizität und Herzschlagvolumen. Zusätzliche Risikofaktoren für Erkrankungen sind Übergewicht sowie Lebensstilfaktoren, etwa Rauchen. Die Kombination aus diesen Risikofaktoren und den generell auftretenden altersphysiologischen Prozessen kann dann im hohen Alter zu funktionalen Einschränkungen führen. Obwohl aber steigendes Alter mit einer sinkenden funktionalen Gesundheit zusammenhängt, ist Hilfe- und Pflegebedürftigkeit, eine Konsequenz sehr eingeschränkter funktionaler Gesundheit, erst im hohen Alter stark verbreitet, in der Regel erst jenseits des 80. Lebensjahres (Menning \& Hoffmann 2009).

Die subjektive Gesundheit ist die individuelle Bewertung des eigenen Gesundheitszustandes und gibt an, wie sich Menschen gesundheitlich fühlen und ihre Einschränkungen und Krankheit(en) selbst einschätzen. Diese Gesundheitsdimension ist insbesondere deshalb von Bedeutung, weil Personen, die sich subjektiv gesund fühlen, im Durchschnitt länger leben als Personen mit einer weniger positiven subjektiven Gesundheitseinschätzung - unabhängig von ihrer körperlichen Gesundheit (Idler \& Benyamini 1997; Benyamini \& Idler 1999). Eine Erklärung dafür könnte sein, dass Personen mit subjektiv besserer Gesundheit eher gesundheitsförderliche Maßnahmen ergreifen, beziehungsweise Risikoverhaltensweisen nicht zeigen oder ändern (Benyamini 2011).

Es kann zu großen Diskrepanzen zwischen objektivem und subjektivem Gesundheitszustand kommen (Chipperfield 1993; Wettstein, Schilling, \& Wahl 2016), besonders im hohen und sehr hohen Alter. Dies zeigt sich unter anderem darin, dass viele ältere Menschen, obwohl es ihnen körperlich und funktional immer schlechter geht, aussagen, dass sie sich gesund fühlen (Spuling, Wurm, Wolff, \& Wünsche 2017a). Zwar nimmt auch die subjektive Bewertung der Gesundheit in der zwei- ten Lebenshälfte $a b$, allerdings weniger stark als es die tatsächliche Verschlechterung der körperlichen Gesundheit vermuten lassen würde (Jylhä, Guralnik, Balfour, \& Fried 2001; Wurm, Lampert, \& Menning 2009). Wenn Menschen ihre eigene Gesundheit bewerten, fließen dabei auch Faktoren jenseits der körperlichen und funktionalen Gesundheit ein, zum Beispiel das Wohlbefinden und die Lebenssituation, das Gesundheitsverhalten, die psychische Gesundheit, die individuelle Persönlichkeit und der eigene Lebensstil (Jylhä 2009). Somit ist es besonders wichtig, auch die selbsteingeschätzte Gesundheit zu berücksichtigen, um ein möglichst umfassendes Bild von der Gesundheitsentwicklung über die zweite Lebenshälfte zu bekommen.

\section{Geschlechterunterschiede in der funktionalen und subjektiven Gesundheit}

Die gesundheitliche Situation von Frauen und Männern unterscheidet sich auf vielfältige Weise. Frauen berichten durchschnittlich mehr funktionale Einschränkungen als Männer (Wolff et al. 2017). Dies kann unter anderem daran liegen, dass Frauen häufiger von Krankheiten betroffen sind, welche die Mobilität beeinflussen, zum Beispiel Arthrose und Osteoporose (Pietschmann, Rauner, Sipos, \& Kerschan-Schindl 2009). Insgesamt erleben Frauen in ihrem Lebenslauf zudem größere physiologische und hormonelle Veränderungen als Männer, verursacht unter anderem durch Schwangerschaft und Menopause. Frauen sind häufiger von Multimorbidität betroffen als Männer (Fuchs, Busch, Lange, \& Scheidt-Nave 2012), was ihr Risiko für funktionale Einschränkungen erhöht. Zudem haben auch die im Durchschnitt geringeren Bildungs- und Einkommensniveaus von Frauen aus älteren Geburtsjahrgängen einen Einfluss auf ihre Gesundheitsentwicklung. Es hat sich gezeigt, dass Gruppen mit niedrigem Einkommen und geringerer Bildung weniger Ressourcen zur Aufrechterhaltung der Gesundheit haben (z. B. finanzielle Mittel, soziale Beziehungen, Wissen über Gesund- 
heit und Gesundheitsverhalten; Lynch 2003). Diese Geschlechterunterschiede in der Gesundheit können also durchaus in geschlechtsspezifischen sozialen Ungleichheiten begründet liegen.

Ergebnisse des Deutschen Alterssurveys (DEAS) zeigen, dass Frauen und Männer ihre Gesundheit subjektiv gleich bewerten, trotz der genannten Unterschiede in der funktionalen Gesundheit (Spuling et al. 2017a). Dies könnte an sozialen Vergleichsprozessen und der dabei von Frauen und Männern genutzten Referenzgruppen liegen: Wenn sich Frauen mit anderen Frauen ihres Alters vergleichen, ist ein gewisses Maß an funktionalen Einschränkungen „normal“ - was so zu einem besseren selbsteingeschätztem Gesundheitszustand führt als wenn sie sich mit gleichaltrigen Männern vergleichen würden (Zajacova, Huzurbazar, \& Todd 2017).

\section{Kohortenunterschiede in der funktionalen und subjektiven Gesundheit von Frauen und Männern}

Geschlechterunterschiede in der Gesundheit müssen jedoch immer in den historischen Kontext eingebettet werden. Die Rolle der Geburtskohorte sollte nicht vernachlässigt werden, da sich im Laufe der letzten Jahrzehnte die Geschlechterverhältnisse in Deutschland und damit auch die gesellschaftliche Stellung der Frau gewandelt haben - Frauen später geborener Kohorten sind besser gebildet und beziehen höhere Einkommen als Frauen früher geborener Kohorten. Die Unterschiede zu den Männern werden hier geringer, so dass in nachfolgenden Kohorten auch Geschlechterunterschiede in der Gesundheit möglicherweise verschwinden oder zumindest geringer ausfallen werden (Crimmins \& Beltrán-Sánchez 2011).

Nicht nur auf Geschlechterunterschiede bezogen, sondern auch generell gibt es historische Entwicklungen, die besonders für das Gesundheitssystem, die Pflegeversicherung und Rentenpolitik wichtige Schlüsse zulassen. Insgesamt ist mit einer Verbesserung des allgemeinen Gesundheits- zustandes zu rechnen, da nachfolgende Kohorten die positiven Auswirkungen des medizinischen Fortschritts, höherer Bildungsniveaus und eines teilweise gesünderen Lebensstils im Allgemeinen $\mathrm{zu}$ spüren bekommen. Zum Beispiel funktioniert heutzutage die Früherkennung vieler Krankheiten sehr gut und insgesamt treiben Menschen mehr Sport. Es konnte anhand von Daten des Deutschen Alterssurveys gezeigt werden, dass in älteren Altersgruppen die sportliche Betätigung zwischen 2008 und 2014 anstieg und dieser positive Wandel bei Frauen besonders stark ausgeprägt war (Spuling, Ziegelmann, \& Wünsche 2017b).

In der Tat wird eine Zunahme der in guter Gesundheit verbrachten Lebensjahre erwartet (Kroll \& Ziese 2009). Weiterhin zeigen Studien, die Daten des Sozio-oekonomischen Panels nutzen, dass später geborene Kohorten weniger funktionale Einschränkungen berichten als früher geborene Kohorten (Trachte, Sperlich, \& Geyer 2015).

In Bezug auf ausschließlich die subjektive Gesundheit sind bisherige Forschungsergebnisse weniger eindeutig und komplexer, was unter anderem auf die unterschiedlichen Einflussfaktoren, die betrachtet wurden, zurückzuführen ist. Dabei finden sich Studien, die eine schlechtere subjektive Gesundheit sowohl bei später Geborenen (Chen, Cohen, \& Kasen 2007; Idler 1993), als auch bei früher Geborenen zeigen (Dening, Chi, Brayne, Huppert, Paykel, \& O'Connor 1998; Heikkinen, Kauppinen, Rantanen, Leinonen, Lyyra, Suutama, \& Heikkinen 2011), sowie Studien, die keine Kohorteneffekte finden (Hoeymans, Feskens, van den Bos, \& Kromhout 1997).

Neben dem Vergleich von Frauen und Männern hinsichtlich der Alternsverläufe ihrer funktionalen und subjektiven Gesundheit ist es also auch wichtig, diese Geschlechterunterschiede im historischen Kontext $\mathrm{zu}$ betrachten, indem die Alternsverläufe von Frauen und Männern unterschiedlicher Geburtskohorten miteinander verglichen werden. Dadurch lässt sich eine Aussage darüber treffen, ob sich im Zuge des gesellschaftlichen Wandels die Alternsverläufe in der Gesundheit von Frauen und Männern einander angenähert haben, 
oder ob die Geschlechterunterschiede in den Verläufen stabil geblieben oder sogar größer geworden sind.

\section{Fragestellungen}

Zusammenfassend werden im vorliegenden Kapitel die folgenden Forschungsfragen untersucht:

\subsection{Methoden}

\section{Stichprobe}

Für das vorliegende Kapitel wurden die Daten aller Erhebungen des Deutschen Alterssurveys (DEAS; 1996, 2002, 2008, 2011, 2014, 2017) berücksichtigt. Insgesamt gehen in die Analysen zur funktionalen Gesundheit (erstmals 2002 erfasst) 15131 Personen ein. Die Analysemodelle zur subjektiven Gesundheit (erstmals 1996 erfasst) berücksichtigen dagegen 20017 Personen.

\section{Indikatoren}

Funktionale Gesundheit. Die funktionale Gesundheit wird mit Hilfe von zehn Fragen zu Einschränkungen in täglichen Aktivitäten (Subskala körperliche Funktionsfähigkeit der SF-36-Skala zu gesundheitsbezogener Lebensqualität; Bullinger \& Kirchberger 1998) im Rahmen des persönlichen Interviews erfasst. Die Befragten werden dazu aufgefordert, anhand einer dreistufigen Rating-Skala anzugeben, ob sie bei den folgenden Alltagstätigkeiten durch ihren derzeitigen Gesundheitszustand „stark eingeschränkt", „etwas eingeschränkt“ oder „überhaupt nicht eingeschränkt" sind: Anstrengende Tätigkeiten (z.B. schnell laufen, schwere Gegenstände heben, anstrengenden Sport treiben); mittelschwere Tätigkeiten (z.B. einen Tisch verschieben, staubsaugen, kegeln, Golf spielen); Einkaufstaschen heben oder tragen; mehrere Trep-
1) Unterscheiden sich Frauen und Männer hinsichtlich ihrer Alternsverläufe funktionaler und subjektiver Gesundheit?

2) Verändern sich Geschlechterunterschiede in den Alternsverläufen funktionaler und subjektiver Gesundheit über verschiedene Geburtskohorten hinweg?

penabsätze steigen; sich beugen, knien, bücken; mehr als einen Kilometer zu Fuß gehen; mehrere Straßenkreuzungen weit zu Fuß gehen; eine Straßenkreuzung weit zu Fuß gehen; sich baden oder anziehen. Die Antworten wurden über jede Person summiert und so transformiert, dass die SkalaAusprägungen zwischen null und 100 liegt, wobei höhere Werte eine geringere Einschränkung und somit eine bessere funktionale Gesundheit bedeuten. In Anlehnung an Nejati und Kollegen (2008) wird bei der Ergebnisdarstellung folgende Kategorisierung als Interpretationshilfe herangezogen: Sehr gute funktionale Gesundheit (Werte 80-100), gute funktionale Gesundheit (60-79), moderate funktionale Gesundheit (40-59), schlechte funktionale Gesundheit (20-39), sehr schlechte funktionale Gesundheit (0-19).

Subjektive Gesundheit. Die subjektive Gesundheit wird im Rahmen des persönlichen Interviews erfasst. Alle Befragten werden aufgefordert, ihren derzeitigen Gesundheitszustand auf einer fünfstufigen Rating-Skala zu bewerten. Die vorgegebenen Antwortalternativen lauteten dabei „sehr gut“, „gut“, „mittel“, „schlecht“, „sehr schlecht“. Höhere Werte - auf einer Skala von 1 „sehr schlecht“ bis 5 ,sehr gut“ - entsprechen einer besseren subjektiven Gesundheit.

Gruppierungsvariablen. Alternsverläufe funktionaler und subjektiver Gesundheit werden zwischen Frauen und Männern sowie zwischen verschiedenen Geburtskohorten verglichen. Zur Beschrei- 
bung von Kohortenunterschieden werden drei ausgewählte Geburtskohorten betrachtet: 1930-1939 Geborene, 1940-1949 Geborene, 1950-1959 Geborene. Diese Auswahl führt dazu, dass sich die Stichprobengröße bei den Analysen zu Kohortenunterschieden auf 10677 Personen bei der funktionalen Gesundheit verringert und auf 13929 Personen bei der subjektiven Gesundheit.

Kontrollvariablen. Als zusätzliche Kontrollvariable dient in allen Analysen die Region (West-/Ostdeutschland) als zentrale Stichprobenvariable des Deutschen Alterssurveys.

\section{Statistische Analyseverfahren}

Mögliche Verzerrungen der Analysen, die sich aus dem selektiven Ausfall von Personen im Längsschnitt ergeben könnten, wurden mit der Full Information Maximum Likelihood Methode ausgeglichen. In die Analysen konnten dadurch auch jene Personen einbezogen werden, die nur einmal am Deutschen Alterssurvey teilgenommen haben. Die Alternsverläufe von funktionaler und sub-

\subsection{Ergebnisse}

\subsubsection{Funktionale Gesundheit im Verlauf der zweiten Lebenshälfte}

Alternsverläufe der funktionalen Gesundheit unterscheiden sich zwischen Frauen und Männern: Frauen berichten über die gesamte zweite Lebenshälfte im Durchschnitt mehr Einschränkungen als Männer.

Die funktionale Gesundheit wird mit zunehmendem Alter im Durchschnitt schlechter. Das heißt funktionale Einschränkungen nehmen zu, je älter Frauen und Männer werden. Insgesamt zeigen sich Geschlechterunterschiede - sowohl im Ausgangsniveau, das wir im Alter von Anfang 40 Jahren betrachten, als auch hinsichtlich der Abnahme jektiver Gesundheit wurden mit Hilfe von Dual Change Score Modellen (McArdle 2001; McArdle \& Hamagami 2001) untersucht. In solchen Modellen werden individuelle Alternsverläufe geschätzt und man kann Unterschiede im Ausgangsniveau, also hier im Alter von 40 Jahren, sowie Unterschiede im Ausmaß von Veränderungen, also hier im Alter zwischen 40 und 90 Jahren, untersuchen und beschreiben. Für diese Veränderung gibt es einen zu schätzenden linearen sowie einen nichtlinearen Anteil.

Ob sich Frauen und Männer hinsichtlich der Alternsverläufe funktionaler und subjektiver Gesundheit unterscheiden, wurde untersucht, indem der Effekt der Variable Geschlecht auf Ausgangsniveau und Veränderung von subjektiver und funktionaler Gesundheit auf Signifikanz getestet wurde. Um Geschlechterunterschiede hinsichtlich der Kohortenunterschiede zu untersuchen, wurde zusätzlich der Effekt der Interaktion zwischen den Variablen Kohorte und Geschlecht auf Ausgangsniveau und Veränderung auf Signifikanz getestet. Das Signifikanzniveau wurde bei allen Analysen auf $\mathrm{p}<0,05$ festgesetzt.

der funktionalen Gesundheit über die zweite Lebenshälfte hinweg. Wie Abbildung 3-1 zeigt, berichten Frauen über den insgesamt betrachteten Alternsverlauf von gut 50 Jahren eine schlechtere funktionale Gesundheit als Männer. Dieser Geschlechterunterschied nimmt mit steigendem Alter zu: Während sich Frauen und Männer im Alter von 40 Jahren nur gering in ihrer funktionalen $\mathrm{Ge}$ sundheit unterscheiden (geschätzte mittlere Differenz auf der Skala von null bis 100: 1,6 Punkte), nehmen funktionale Einschränkungen bei Frauen mit zunehmendem Alter sehr viel stärker zu als es bei Männern der Fall ist (geschätzte mittlere Differenz am Ende des achten Lebensjahrzehnts: rund 13 Punkte). Diese stärkere Abnahme der funktionalen Gesundheit führt dazu, dass sich Frauen und 
Abbildung 3-1 Funktionale Gesundheit bei Frauen und Männern im Alternsverlauf

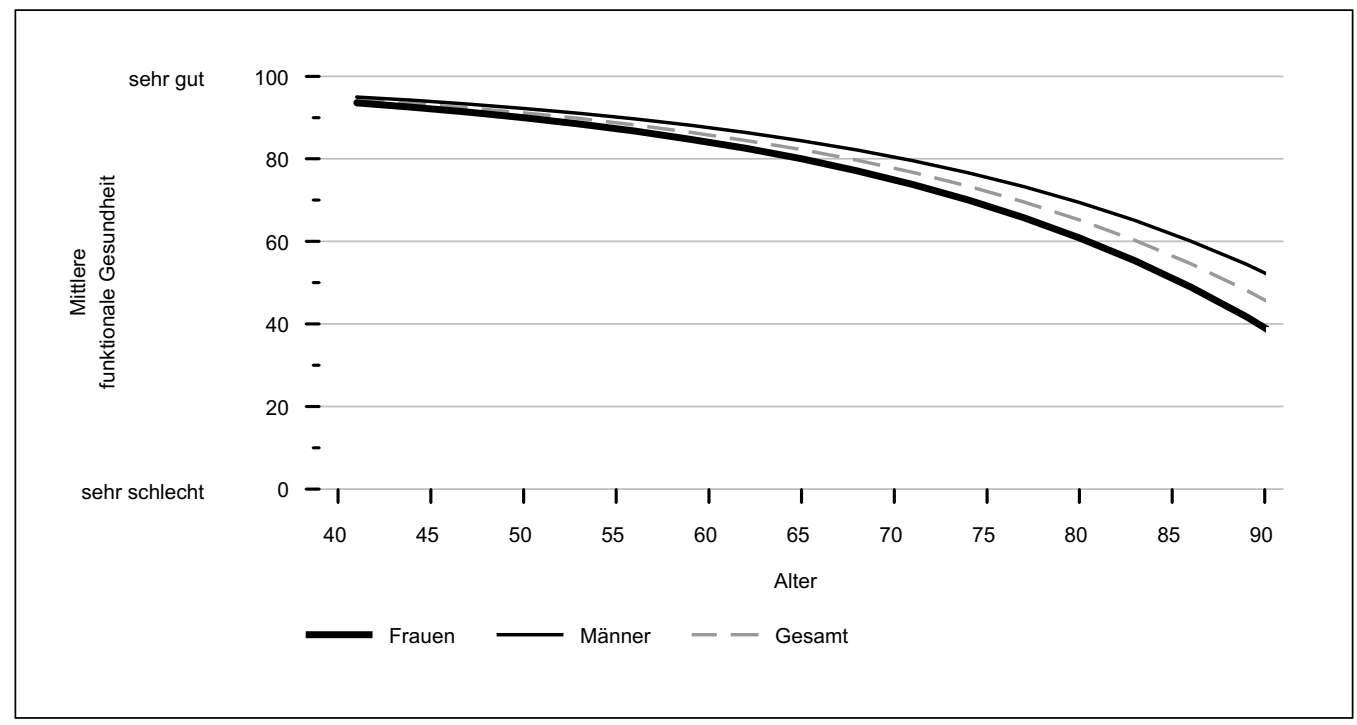

Quelle: DEAS 2002-2017, Schätzungen basieren auf $n=15131$ Studienteilnehmenden mit insgesamt $n=28882$ Beobachtungen; kontrolliert für Region; Kategorisierung der Werte nach Nejati et al. (2008): sehr gute funktionale Gesundheit (80-100), gute funktionale Gesundheit (60-79), moderate funktionale Gesundheit (40-59), schlechte funktionale Gesundheit (20-39), sehr schlechte funktionale Gesundheit (o-19).

Unterschiede zwischen Frauen und Männern im geschätzten Ausgangsniveau (Alter Anfang 40 Jahre) sind signifikant - ebenso unterscheidet sich der geschätzte Verlauf zwischen Frauen und Männern signifikant. Frauen haben im Durchschnitt eine etwas schlechtere funktionale Gesundheit im Alter von 40 Jahren und die Abnahme mit zunehmendem Alter fällt bei Frauen im Durchschnitt stärker aus als bei Männern. Folglich vergrößern sich Geschlechterunterschiede hinsichtlich der funktionalen Gesundheit mit zunehmendem Alter.

Männer im hohen Alter sehr viel stärker in ihrer funktionalen Gesundheit unterscheiden als es im mittleren Erwachsenenalter der Fall ist. Bei Frauen wechselt ihre durchschnittliche funktionale Gesundheit ab einem Alter von ca. 65 Jahren vom Bereich „sehr gut" zu "gut" und ab einem Alter von ca. 81 Jahren von "gut" (Werte zwischen 60 und 79) zu „moderat“ (Werte zwischen 40 und 59). Bei Männern erfolgen diese Wechsel jeweils später im Alternsverlauf: Von „sehr gut“ zu „gut“ (Werte zwischen 60 und 79) durchschnittlich in einem Alter von etwa 70 Jahren, und von "gut“ zu „moderat" durchschnittlich in einem Alter von etwa 86 Jahren. Die beschriebenen Übergänge in der funktionalen Gesundheit finden bei Männern im Durchschnitt also jeweils ca. fünf Jahre später statt als bei Frauen. Männer erreichen im Durchschnitt auch im sehr hohen Alter nicht den Wertebereich von „schlechter" funktionaler Gesundheit (Werte zwischen 20 und 39), und bei Frauen vollzieht sich dieser Übergang erst im Übergang vom 89. zum 90. Lebensjahr. Die durchschnittliche funktionale Gesundheit der Frauen sinkt also selbst in diesem sehr hohen Alter nicht in den Bereich „sehr schlecht" (Werte zwischen 0 und 19), und bei den Männern ist sie im Durchschnitt auch im sehr hohen Alter noch „moderat“.

Für die funktionale Gesundheit zeigen sich Kohortenunterschiede: Frauen und Männer später geborener Kohorten werden vermutlich mit einer besseren funktionalen Gesundheit ins hohe und sehr hohe Al- 
ter kommen als Frauen und Männer früher geborener Kohorten.

Sowohl im geschätzten durchschnittlichen Ausgangsniveau im mittleren Erwachsenenalter als auch hinsichtlich der Stärke der durchschnittlichen Abnahme mit zunehmendem Alter zeigen sich Kohortenunterschiede in der funktionalen Gesundheit (Abbildung 3-2). Während im mittleren Erwachsenenalter die früher geborene Kohorte (in den Jahren 1930-1939 Geborene) im Durchschnitt eine bessere funktionale Gesundheit im Sinne weniger berichteter Einschränkungen aufweist als die beiden später geborenen Kohorten (gemäß unserer Modellschätzung, denn die Gesundheit der 1930 bis 1939 Geborenen wurde im
Alter von 40 Jahren im Rahmen des Deutschen Alterssurveys (DEAS) nicht erfasst), ist die Abnahme bis zum Ende des achten Lebensjahrzehnts in dieser Kohorte am stärksten ausgeprägt. Mit anderen Worten: Der Vorteil früherer Geburtskohorten gegenüber später Geborenen hinsichtlich der funktionalen Gesundheit ist also nur im mittleren Erwachsenenalter zu beobachten und dreht sich dann im Alter von ca. 70 Jahren zugunsten späterer Geburtsjahrgänge um. Die am spätesten geborene hier betrachtete Kohorte (1950-1959 Geborene) hat im mittleren Erwachsenenalter eine etwas schlechtere funktionale Gesundheit als die beiden anderen Geburtskohorten, verzeichnet jedoch eine geringere Abnahme der funktionalen Gesundheit mit zunehmendem Alter im Vergleich zu 1940 bis

\section{Abbildung 3-2 Funktionale Gesundheit nach Kohorten im Alternsverlauf}

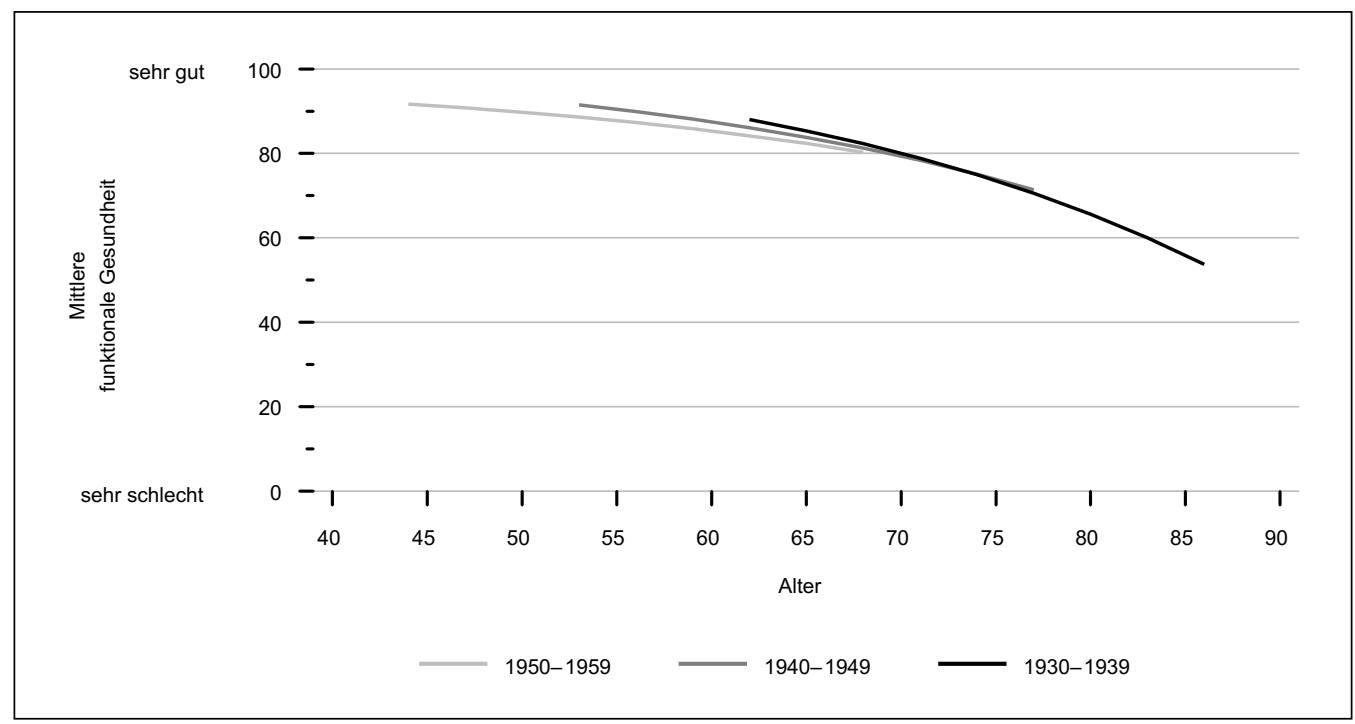

Quelle: DEAS 2002-2017, Schätzungen basieren auf $n=10677$ Studienteilnehmenden mit insgesamt $n=21146$ Beobachtungen; kontrolliert für Region und Geschlecht; der geschätzte Alternsverlauf für die funktionale Gesundheit in der Geburtskohorte 1950-1959 beginnt erst ab einem Alter von 43 Jahren, da die funktionale Gesundheit erst seit 2002 Bestandteil des DEAS-Instruments ist; Kategorisierung der Werte nach Nejati et al. (2008): sehr gute funktionale Gesundheit (80-100), gute funktionale Gesundheit (60-79), moderate funktionale Gesundheit (40-59), schlechte funktionale Gesundheit (20-39), sehr schlechte funktionale Gesundheit (o-19).

Unterschiede zwischen den Geburtskohorten im geschätzten Ausgangsniveau (Alter Anfang 40 Jahre) sind signifikant - ebenso unterscheidet sich der geschätzte Verlauf zwischen den Geburtskohorten signifikant. Später Geborene haben eine schlechtere funktionale Gesundheit im mittleren Erwachsenenalter im Vergleich zu früher Geborenen (wenn man den Verlauf der früher geborenen Kohorten bis ins mittlere Erwachsenenalter weiterführt), dafür fällt die Abnahme mit zunehmendem Alter bei später Geborenen schwächer aus als bei früher Geborenen. 
1949 und 1930 bis 1939 Geborenen. Es kann folglich erwartet werden, dass die später geborenen Kohorten mit einer besseren funktionalen Gesundheit im Sinne weniger berichteter funktionaler Einschränkungen ins hohe und sehr hohe Alter kommen werden als früher geborene Kohorten. Diese Kohortenunterschiede treffen gleichermaßen auf Frauen und Männer zu (vgl. Abbildung 3-3).

Dieser Kohorteneffekt trifft gleichermaßen auf Frauen und Männer zu. Das heißt, die Abnahme der funktionalen Gesundheit mit zunehmendem Alter fällt in früher geborenen Kohorten gleichermaßen bei Frauen und Männern stärker aus als es bei Frauen und Männern später geborener Kohorten der Fall ist, und die Geschlechterdiskrepanz im Verlauf unterscheidet sich nicht signifikant zwischen den Kohorten.

\subsubsection{Subjektive Gesundheit im Verlauf der zweiten Lebenshälfte}

Alternsverläufe der subjektiven Gesundheit unterscheiden sich nicht zwischen Frauen und Männern: Frauen und Männer bewerten gleichermaßen ihre Gesundheit mit zunehmendem Alter schlechter.

Sowohl im Ausgangsniveau (im mittleren Erwachsenenalter) als auch in der Veränderung der subjektiven Gesundheit im Verlauf der zweiten Lebenshälfte unterscheiden sich Frauen und Männer nicht. In Abbildung 3-4 ist der allgemeine durchschnittliche Alternsverlauf der subjektiven Gesundheit verdeutlicht sowie der durchschnittliche Alternsverlauf für Frauen und Männer getrennt. Wie in Abbildung 3-4 dargestellt, überlappen sich die drei Linien. Das heißt, Frauen und Männer unterscheiden sich weder im mittleren Erwachsenenalter, noch wenn sie älter werden, signifikant in ihrer subjektiven Gesundheitsbewertung. Im mittleren Erwachsenenalter (Anfang des fünften Lebensjahrzehnts) bewerten Menschen ihre Gesundheit auf der fünfstufigen Antwortskala im Durchschnitt mit dem Wert 3,8 („gut“); wenn Frauen und Männer das Ende des neunten Lebens- jahrzehnts und somit das sehr hohe Alter erreichen, liegt der durchschnittliche Wert ihrer subjektiven Gesundheitsbewertung immer noch bei 2,9 („mittel“).

Für die subjektive Gesundheit zeigen sich keine Kohortenunterschiede: Frauen und Männer späterer Geburtsjahrgänge unterscheiden sich nicht im Ausgangsniveau und im Alternsverlauf ihrer Gesundheitsbewertung von Frauen und Männern früherer Geburtsjahrgänge.

Hinsichtlich der subjektiven Gesundheit sind keine Kohortenunterschiede beobachtbar - weder bei Frauen noch bei Männern (Abbildung 3-5). Der durchschnittliche Alternsverlauf der subjektiven Gesundheit getrennt für die drei betrachteten Geburtskohorten verläuft ähnlich wie der allgemeine durchschnittliche Verlauf der subjektiven Gesundheit (Abbildung 3-4). Der allgemeine durchschnittliche Alternsverlauf der subjektiven Gesundheit trifft also nicht nur gleichermaßen auf Frauen und Männer zu, sondern auch gleichermaßen auf alle drei betrachteten Geburtskohorten (1930-1939 Geborene; 1940-1949 Geborene; 19501959 Geborene). 
Abbildung 3-3 Funktionale Gesundheit bei Frauen und Männern nach Kohorten im Alternsverlauf

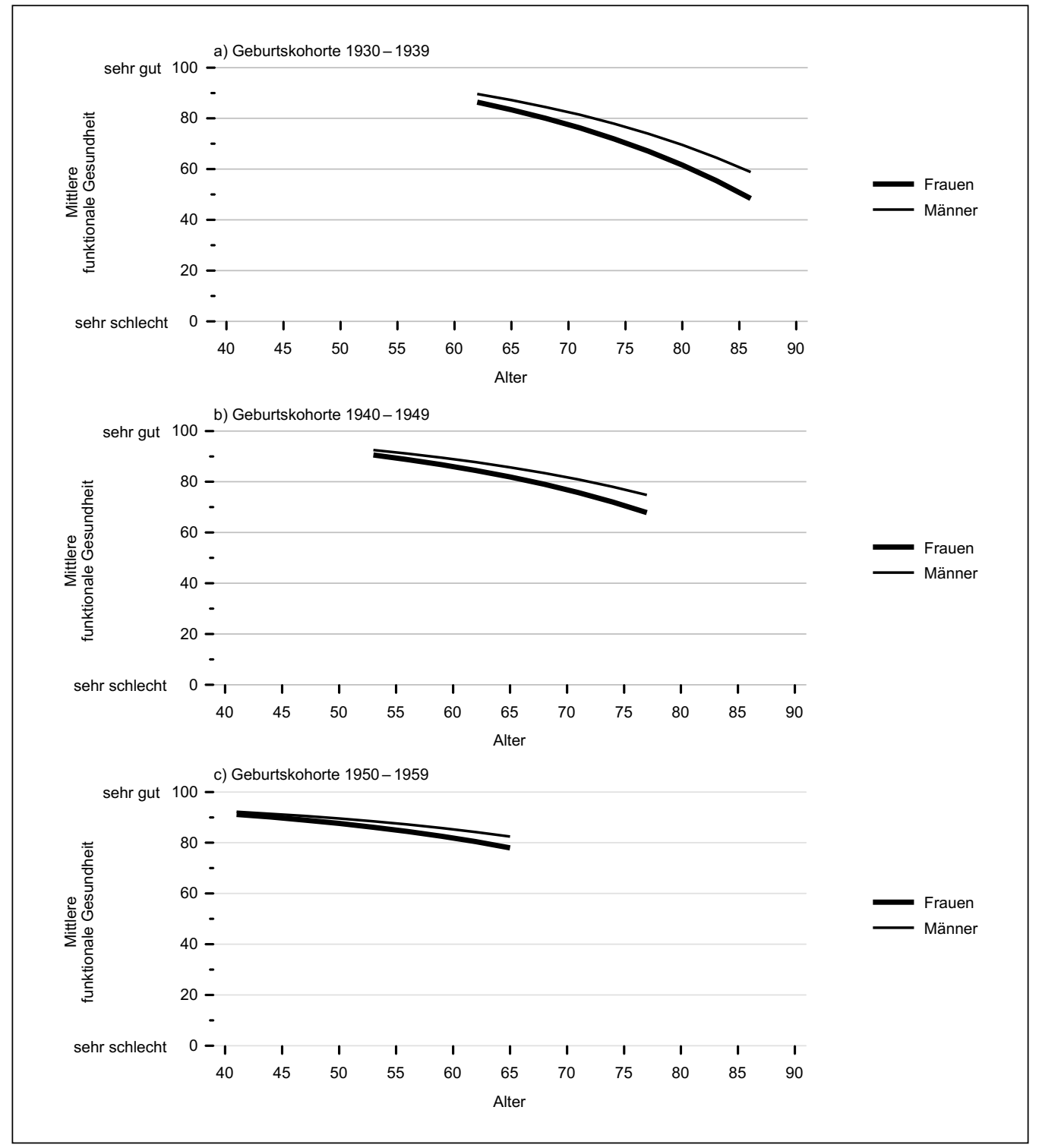

Quelle: DEAS 2002-2017, Schätzungen basieren auf $n=10677$ Studienteilnehmenden mit insgesamt $n=21146$ Beobachtungen; kontrolliert für Region; die geschätzten Alternsverläufe für die funktionale Gesundheit in der Geburtskohorte 1950-1959 beginnen erst ab einem Alter von 43 Jahren, da die funktionale Gesundheit erst seit 2002 Bestandteil des DEAS-Instruments sind; Kategorisierung der Werte nach Nejati et al. (2008): sehr gute funktionale Gesundheit (80-100), gute funktionale Gesundheit (6o-79), moderate funktionale Gesundheit (40-59), schlechte funktionale Gesundheit (20-39), sehr schlechte funktionale Gesundheit (o-19).

Kohortenunterschiede hinsichtlich der funktionalen Gesundheit treffen auf Frauen und Männer gleichermaßen zu: Die Abnahme der funktionalen Gesundheit mit steigendem Alter fällt bei später geborenen Frauen und Männern schwächer aus als bei früher geborenen Frauen und Männern. Geschlechterunterschiede zeigen sich gleichermaßen in allen drei betrachteten Kohorten. 
Abbildung 3-4 Subjektive Gesundheit bei Frauen und Männern im Alternsverlauf

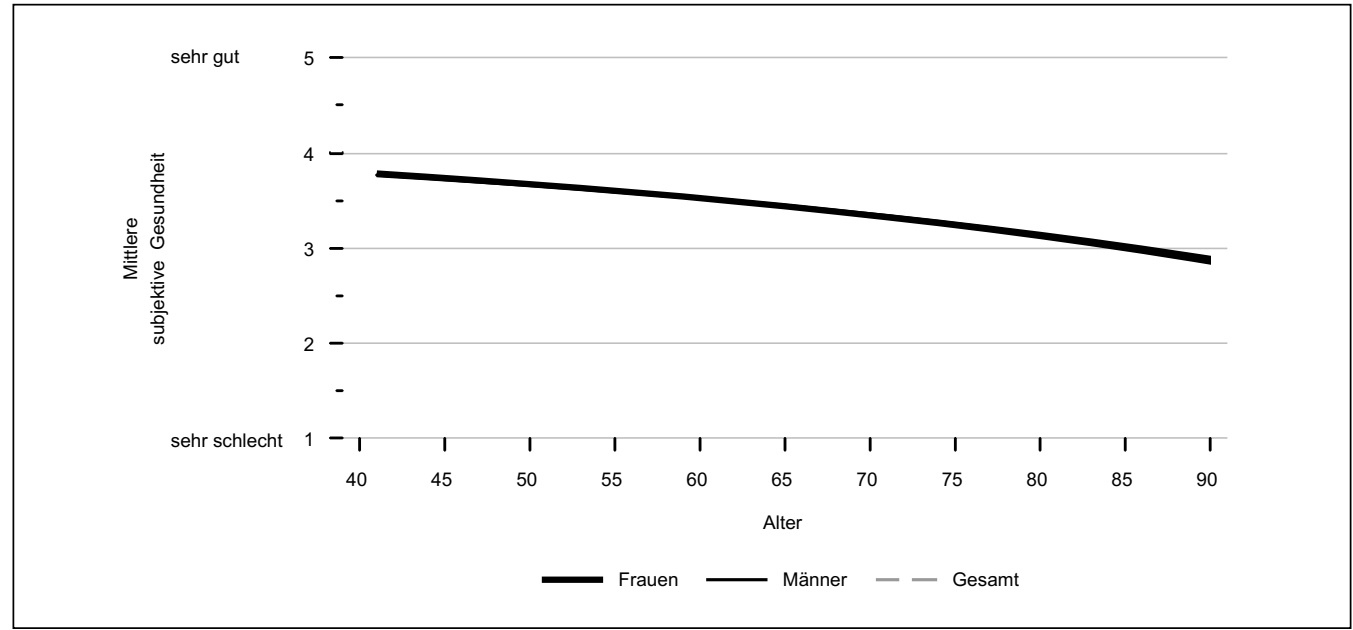

Quelle: DEAS 1996-2017, Schätzungen basieren auf $\mathrm{n}=20017$ Studienteilnehmenden mit insgesamt $\mathrm{n}=38893$ Beobachtungen; kontrolliert für Region und Geschlecht; 1 („sehr schlechte subjektive Gesundheit“), 2 („schlechte subjektive Gesundheit“), 3 („mittlere subjektive Gesundheit“), 4 („gute subjektive Gesundheit“), 5 („sehr gute subjektive Gesundheit“).

Unterschiede zwischen Frauen und Männern hinsichtlich ihrer subjektiven Gesundheit im geschätzten Ausgangsniveau (Alter Anfang 40 Jahre) und im geschätzten Verlauf sind statistisch nicht signifikant. Die Abnahme der subjektiven Gesundheit mit zunehmendem Alter erfolgt für Frauen und Männer gleichermaßen nicht linear.

\section{Abbildung 3-5 Subjektive Gesundheit nach Kohorten im Alternsverlauf}

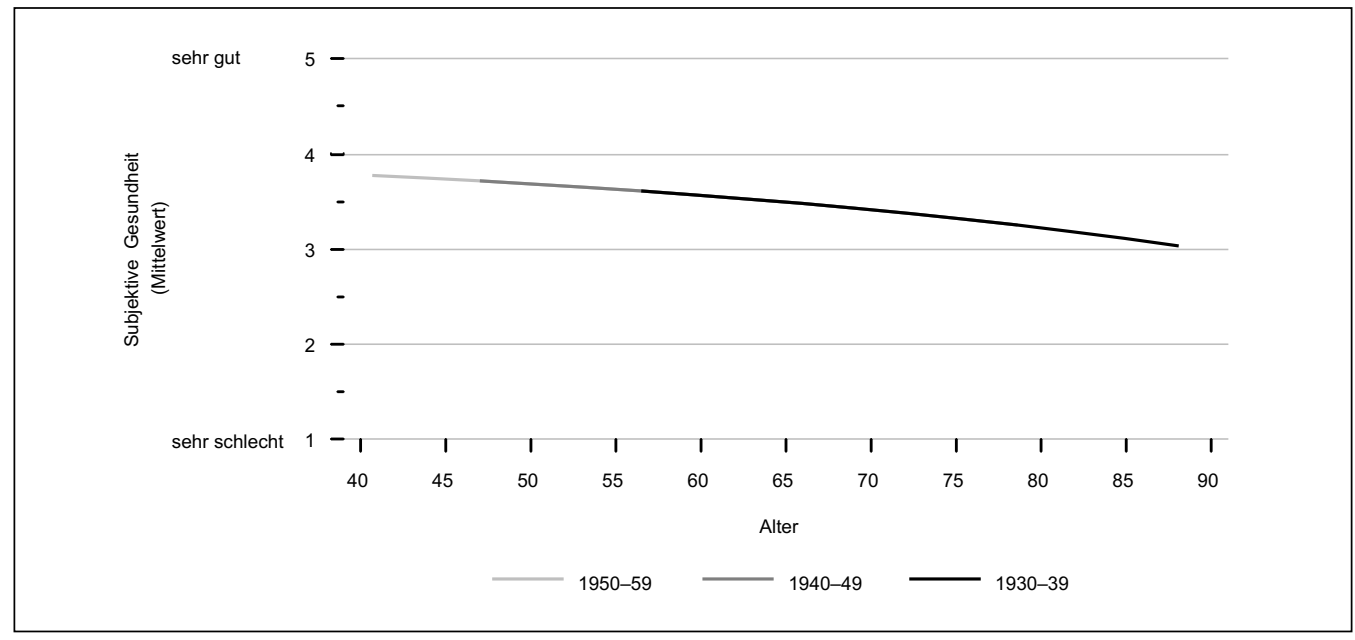

Quelle: DEAS 1996-2017, Schätzungen basieren auf $n=13929$ Studienteilnehmenden mit insgesamt $\mathrm{n}=28887$ Beobachtungen; kontrolliert für Region und Geschlecht; 1 („sehr schlechte subjektive Gesundheit“), 2 („,schlechte subjektive Gesundheit“), 3 („mittlere subjektive Gesundheit“), 4 („gute subjektive Gesundheit“), 5 („sehr gute subjektive Gesundheit“).

Unterschiede zwischen den Geburtskohorten im geschätzten Ausgangsniveau (Alter Anfang 40 Jahre) sowie im geschätzten Verlauf sind nicht signifikant. 


\subsection{Diskussion}

\section{Zusammenfassung}

Im Allgemeinen nehmen sowohl die funktionale als auch die subjektive Gesundheit der Frauen und Männer über die zweite Lebenshälfte ab. Geschlechterunterschiede zeigen sich in diesem $\mathrm{Zu}$ sammenhang ausschließlich bei Betrachtung der Alternsverläufe der funktionalen Gesundheit: Sowohl Ausgangsniveau als auch Abnahme mit zunehmendem Alter fallen für Frauen negativer aus als für Männer. Weiterhin gibt es Kohortenunterschiede in den Alternsverläufen der funktionalen Gesundheit, die auf Frauen und Männer gleichermaßen zutreffen: Früher geborene Kohorten haben eine bessere funktionale Gesundheit im mittleren Erwachsenenalter als später geborene Kohorten. Später geborene Kohorten weisen dagegen eine schwächere Abnahme der funktionalen Gesundheit mit zunehmendem Alter auf und für sie wird deshalb eine bessere funktionale $\mathrm{Ge}$ sundheit im späten Erwachsenenalter erwartet als bei früher geborenen Kohorten. In der Einschätzung ihrer subjektiven Gesundheit und in der Veränderung dieser Einschätzung mit zunehmendem Alter unterscheiden sich Frauen und Männer dagegen nicht. Obwohl Frauen also mehr funktionale Einschränkungen berichten als Männer und zusätzlich eine stärkere Abnahme der funktionalen Gesundheit mit zunehmendem Alter erleben als Männer, unterscheiden sie sich nicht von Männern in ihrer subjektiven Gesundheit und deren Veränderung mit zunehmendem Alter.

\section{Interpretation}

Die funktionale Gesundheit geht besonders im höheren Alter deutlich zurück, bei der subjektiven Gesundheit lassen sich dagegen nur leichte Einbußen feststellen. Dies unterstreicht die Multidimensionalität der gesundheitlichen Situation von Personen - Gesundheit umfasst verschiedene Aspekte, die sich im Alternsverlauf unterschied- lich entwickeln können. Ältere Personen tendieren dazu, sich gesundheitlich mit Gleichaltrigen $\mathrm{zu}$ vergleichen (Leinonen, Heikkinen, \& Jylhä 2001), was zu einer subjektiv positiveren Bilanz führt als der Vergleich mit jüngeren Altersgruppen. Ältere Menschen passen zudem ihre eigenen Standards und Erwartungen hinsichtlich ihrer Gesundheit an Veränderungen ihres körperlichen und funktionalen Gesundheitszustandes an, ein Phänomen, das in der Literatur als Response Shift bezeichnet wird (Sprangers \& Schwartz 1999). So wird eine positive subjektive Bewertung der eigenen Gesundheit begünstigt (Galenkamp, Huisman, Braam, \& Deeg 2012; Spuling, Wolff, \& Wurm 2017a). Das bedeutet, dass ältere Menschen im Allgemeinen eine Verschlechterung ihrer Gesundheit erwarten und diese als „normal“ bewerten. Das heißt wiederum, dass eine Verschlechterung des körperlichen oder funktionalen Gesundheitszustandes nicht unbedingt mit einer Veränderung der subjektiven Bewertung der Gesundheit einhergehen muss.

Alternsverläufe funktionaler und subjektiver Gesundheit von Frauen und Männern: Bei Betrachtung der funktionalen Gesundheit zeigen sich Geschlechterunterschiede: Frauen sind gegenüber Männern im Alter von 40 Jahren häufiger von funktionalen Einschränkungen betroffen, sie haben also eine etwas geringere funktionale Gesundheit. Dieser Geschlechterunterschied wird im Laufe des Alterns größer, da Frauen stärkere Einbußen bei der funktionalen Gesundheit erleiden als Männer. Dies kann daran liegen, dass Frauen eine höhere Lebenserwartung haben als Männer und infolge der höheren Lebenserwartung mehr Frauen als Männer trotz gesundheitlicher Einbußen ein hohes Alter erreichen. Die hier beschriebenen Geschlechterunterschiede über die gesamte zweite Lebenshälfte hinweg könnten darüber hinaus eventuell aber auch durch Bildungsunterschiede zwischen Frauen und Männern erklärt werden. Bildung spielt eine wichtige Rolle für körperliche und funktionale Gesundheitsverläufe (Lynch 2003; Schöllgen, Huxhold, \& Tesch-Römer 2010), da eine 
höhere Bildung über unterschiedliche Mechanismen mit einem insgesamt gesundheitsförderlicheren Leben zusammenhängt. Auch geschlechtsspezifische Ungleichheiten in der Arbeitswelt, welche auch in der heutigen Zeit noch fortwähren, können die Unterschiede in der funktionalen Gesundheit von Frauen und Männern möglicherweise erklären. Ein höheres Einkommen, begünstigt durch höhere Bildung, ermöglicht einen gesünderen Lebensstil und eine bessere medizinische Versorgung.

Hinsichtlich der subjektiven Gesundheit zeigt sich kein Geschlechterunterschied - weder im Ausgangsniveau im Alter von 40 Jahren noch im Verlauf des Älterwerdens. Wenn man dabei noch die im Allgemeinen schlechtere psychische Gesundheit von Frauen im Alter (zumindest hinsichtlich depressiver Symptome; siehe Kapitel 4 Lebenszufriedenheit und depressive Symptome in diesem Band) hinzuzieht, ergibt sich ein interessantes Bild - trotz größerer funktionaler Einschränkungen und stärkerer depressiver Symptome, zeigen Frauen gegenüber Männern keinen schlechteren subjektiven Gesundheitszustand und mit zunehmendem Alter keinen stärkeren Rückgang in der subjektiven Gesundheit. Dafür gibt es unterschiedliche Erklärungsansätze: Es kann zum Beispiel sein, dass Frauen ein anderes Gesundheitsverständnis haben als Männer. Das bedeutet, dass bei Frauen möglicherweise zusätzliche oder andere Faktoren in die selbstbeurteilte Gesundheit einfließen beziehungsweise dass bei Frauen die funktionale Gesundheit weniger eng mit der subjektiven Gesundheitsbewertung zusammenhängt als bei Männern. Außerdem ist die subjektive Gesundheit auch stark vom sozialen Vergleichsrahmen abhängig - Frauen und Männer vergleichen ihre eigene Gesundheit nicht nur mit Gleichaltrigen, sondern auch mit Gleichaltrigen desselben Geschlechts (Wills 1992).

Alternsverläufe funktionaler und subjektiver Gesundheit bei verschiedenen Geburtskohorten: Für verschiedene Geburtsjahrgänge zeigen sich unterschiedliche Verläufe in der funktionalen Gesundheit. Früher geborene Kohorten (1930-1939) weisen eine bessere funktionale Gesundheit im mittleren Erwachsenenalter auf als später geborene Kohorten (1940-1949 und 1950-1959). Dafür nimmt die funktionale Gesundheit für die früher geborenen Kohorten aber stärker ab als für die später geborenen Kohorten. Im hohen Alter haben dementsprechend später geborene eine bessere funktionale Gesundheit als früher geborene Jahrgänge. Auch für die Analysen dieses Kapitels gilt dabei, dass sich diese in die Zukunft gerichteten Verlaufsschätzungen für die später geborenen Kohorten - die aktuell noch nicht das (sehr) hohe Alter erreicht haben - als unzutreffend erweisen können. Ihnen liegt die Annahme zugrunde, dass sich die bislang beobachteten Trends zukünftig fortsetzen werden.

Eine mögliche Erklärung für die Unterschiede im mittleren Erwachsenenalter ist hier der sich im Allgemeinen wandelnde Lebensstil: Je später eine Person geboren wird, desto höher ist die Risikowahrscheinlichkeit für Übergewicht und damit verbundene Erkrankungen wie Diabetes oder Herz-Kreislauf-Erkrankungen (Prentice 2006). Zusätzlich scheint das mittlere Erwachsenenalter - gerade in den später geborenen Kohorten - eine belastungsreiche Lebensphase zu sein, die eine potenzielle Gefahr für die sogenannte Work-Life-Balance darstellt. Anforderungen und Stressoren aus unterschiedlichen Lebensbereichen wie Familie und Arbeit treffen aufeinander, wobei Interessenund Zielkonflikte entstehen können (Knecht, Bauer, Gutzwiller, \& Hämmig 2011). Diese Stressoren können sich auf die Gesundheit auswirken. Gleichzeitig haben sich aber auch die medizinischen Möglichkeiten weiterentwickelt, welche den jüngeren Jahrgängen schon einen längeren Teil ihres Lebens zur Verfügung stehen werden als älteren Jahrgängen. Damit können die Folgen einer schlechten funktionalen Gesundheit im mittleren Erwachsenenalter im Hinblick auf die Entwicklung dieser womöglich besser „abgepuffert“ werden als in früher geborenen Kohorten - Erkrankungen werden früher erkannt und erfolgversprechender behandelt, somit werden mögliche funktionale Einschränkungen und eine daraus resultierende 
negativere Gesundheitsentwicklung über die zweite Lebenshälfte vermieden.

Diese Kohortenunterschiede stellen sich sowohl für Frauen als auch für Männer gleichermaßen dar. Dies bedeutet wiederum, dass die bereits herausgestellten Unterschiede zwischen Frauen und Männern in allen untersuchten Geburtskohorten auf ähnliche Weise zum Tragen kommen. Sie sind bislang also nicht im Zuge des gesellschaftlichen Wandels kleiner geworden.

Währenddessen scheint es bei der subjektiven Gesundheit keine Kohortenunterschiede zu geben, das heißt, dass die Abnahme über die Lebensspanne für die betrachteten Geburtsjahrgänge gleich bleibt. Der positive Kohortentrend im Alternsverlauf der funktionalen Gesundheit spiegelt sich also nicht in dem allgemeinen Alternsverlauf der subjektiven Gesundheit wider. Dieses Ergebnis unterstreicht, wie wichtig es ist, verschiedene Bereiche der Gesundheit parallel zu betrachten und zu untersuchen, da sich Veränderungen zwischen den Gesundheitsbereichen unterscheiden können. Dass sich die subjektive Gesundheit im Alternsverlauf nicht signifikant zwischen den Kohorten unterscheidet, könnte daran liegen, dass später geborene Frauen und Männer ein anderes, breiteres Verständnis von Gesundheit haben (z. B. Spuling, Wurm, Tesch-Römer, \& Huxhold 2015) und eventuell höhere Ansprüche an ihre Gesundheit stellen (z. B. Jylhä 2009).

\section{Politische Implikationen}

Für Gesundheitsversorgung und Gesundheitsförderungsmaßnahmen lassen sich aufgrund der Befunde wichtige Schlüsse ziehen. Personen im hohen und sehr hohen Alter gehören allgemein zur Risikogruppe in Bezug auf Einbußen der (funktionalen) Gesundheit (Baltes \& Smith 2003). Eine gute funktionale Gesundheit ist jedoch eine wesentliche Voraussetzung vor allem für Lebensqualität, eine selbstständige Lebensführung aber auch um Aktivitäten nachgehen zu können, die einem Freude bereiten, beispielsweise Zeit mit den En- kelkindern verbringen zu können oder ein Ehrenamt auszuüben. Besonderes Augenmerk sollte hier - neben der Risikogruppe alter und sehr alter Menschen - auf Frauen im Alter gelegt werden. Diese zeigen durchschnittlich eine durchgehend schlechtere funktionale Gesundheit in der zweiten Lebenshälfte sowie eine stärkere Abnahme im Alternsverlauf als Männer. Das heißt, dass Personen im hohen Alter und dabei besonders Frauen von maßgeschneiderten Interventionen profitieren könnten.

Zudem ist der Befund, dass auch in den später geborenen Kohorten der durchschnittliche Alternsverlauf der funktionalen Gesundheit bei Frauen negativer ausfällt als bei Männern, Anlass, über geschlechtsspezifische Interventionsmaßnahmen nachzudenken. Der gesellschaftliche Wandel hat also offenbar nicht oder noch nicht dazu beigetragen, diese Geschlechterdiskrepanz zu reduzieren, so dass es umso wichtiger ist, Maßnahmen zu ergreifen und Rahmenbedingungen zu schaffen, damit nicht auch künftige Kohorten von Frauen mit einer im Vergleich zu Männern eingeschränkteren Gesundheit ins hohe und sehr hohe Alter kommen. Während eine Angleichung von Frauen und Männern im Bereich der Bildung bereits erfolgreich erreicht werden konnte und dies vermutlich positiv auf die Geschlechterunterschiede in der funktionalen Gesundheit künftiger Kohorten wirken wird, ist es zudem wünschenswert, dass noch fortwährende geschlechtsspezifische Differenzen in der Arbeitswelt und in der Vereinbarkeitsproblematik hinsichtlich der Anforderungen aus dem Berufs- und Familienleben ebenfalls angegangen werden, um so eine nachhaltige Verbesserung der funktionalen Gesundheit für Frauen künftiger Kohorten zu erreichen.

Ein weiterer Ansatzpunkt ist die Lebensphase im mittleren Erwachsenenalter: Später geborene Kohorten haben zum einen vermutlich weniger funktionale Einschränkungen im hohen Alter, was unter anderem durch den medizinischen Fortschritt und ein besseres Gesundheitssystem begründet werden kann. Zum anderen zeigen diese später geborenen Kohorten jedoch eine geringe- 
re funktionale Gesundheit im mittleren Erwachsenenalter als früher geborene Kohorten. Dies kann auf einen teilweise ungesünder werdenden Lebensstil (Faktoren wie Übergewicht oder Tabakkonsum), aber auch eine über die Kohorten zunehmende Anhäufung von Stressoren im mittleren Erwachsenenalter, zurückzuführen sein (siehe Kapitel 6 Unbezahlte Sorgetätigkeiten in diesem
Band). Dementsprechend könnten hier Präventionsmaßnahmen zu Gesundheitsverhalten und Stressmanagement gerade für Menschen im mittleren Erwachsenenalter wichtig sein, um funktionalen Einschränkungen und einer Fortsetzung des negativen Kohortentrends in dieser Altersphase entgegenzuwirken.

\section{Literatur}

Baltes, M. M., Maas, I., Wilms, H.-U., \& Borchelt, M. (2010). Alltagskompetenz im Alter: Theoretische Überlegungen und empirische Befunde. In: U. Lindenberger, J. Smith, K. U. Mayer \& P. B. Baltes (Hrsg.) Die Berliner Altersstudie (S. 549-566). Berlin: Akademie-Verlag.

Baltes, P. B., \& Smith, J. (2003). New frontiers in the future of aging: From successful aging of the young old to the dilemmas of the fourth age. Gerontology, 49(2), 123-135.

Barnett, K., Mercer, S. W., Norbury, M., Watt, G., Wyke, S., \& Guthrie, B. (2012). Epidemiology of multimorbidity and implications for health care, research, and medical education: a cross-sectional study. The Lancet, 380(9836), 37-43.

Benyamini, Y. (2011). Why does self-rated health predict mortality? An update on current knowledge and a research agenda for psychologists. Psychology \& Health, 26(11), 1407-1413. doi: 10.1080/08870446.2011.621703.

Benyamini, Y., \& Idler, E. L. (1999). Community studies reporting association between self-rated health and mortality: additional studies, 1995 to 1998. Research on Aging, 21(3), 392-401.

Böhm, K., Tesch-Römer, C., \& Ziese, T. (2009). Gesundheit und Krankheit im Alter [Beiträge zur Gesundheitsberichterstattung des Bundes]. Berlin: Robert Koch-Institut.

Bullinger, M., \& Kirchberger, I. (1998). Der SF-36-Fragebogen zum Gesundheitszustand. Handanweisung. Göttingen, Germany: Hogrefe.

Chen, H., Chen, P., \& Kasen, S. (2007). Cohort differences in self-rated health: evidence from a threedecade, community-based, longitudinal study of women. American Journal of Epidemiology, 166(4), 439-446.

Chipperfield, J. G. (1993). Incongruence between health perceptions and health problems: Implications for survival among seniors. Journal of Aging and Health, 5(4), 475-496.

Crimmins, E. M., \& Beltrán-Sánchez, H. (2011). Mortality and morbidity trends: is there compression of morbidity? The Journals of Gerontology, Series B: Psychological Sciences and Social Sciences, 66(1), $75-86$.

Dening, T. R., Chi, L.-Y., Brayne, C., Huppert, F. A., Paykel, E. S., \& O'Connor, D. W. (1998). Changes in selfrated health, disability and contact with services in a very elderly cohort: a 6-year follow-up study. Age and Ageing, 27(1), 23-33.

Fuchs, J., Busch, M., Lange, C., \& Scheidt-Nave, C. (2012). Prevalence and patterns of morbidity among adults in Germany. Bundesgesundheitsblatt - Gesundheitsforschung - Gesundheitsschutz, 55(4), $576-$ 586. 
Galenkamp, H., Huisman, M., Braam, A. W., \& Deeg, D. J. (2012). Estimates of prospective change in self-rated health in older people were biased owing to potential recalibration response shift. Journal of Clinical Epidemiology, 65(9), 978-988.

Heikkinen, E., Kauppinen, M., Rantanen, T., Leinonen, R., Lyyra, T.-M., Suutama, T., \& Heikkinen, R.-L. (2011). Cohort differences in health, functioning and physical activity in the young-old Finnish population. Aging clinical and experimental research, 23(2), 126-134.

Hoeymans, N., Feskens, E., Bos, G. van den, \& Kromhout, D. (1997). Age, time, and cohort effects on functional status and self-rated health in elderly men. American Journal of Public Health, 87(10), 16201625.

Idler, E. L. (1993). Age differences in self-assessments of health: Age changes, cohort differences, or survivorship? Journal of Gerontology, 48(6), S289-S300.

Idler, E. L., \& Benyamini, Y. (1997). Self-rated health and mortality: a review of twenty-seven community studies. Journal of Health and Social Behavior, 38(1), 21-37.

Jylhä, M. (2009). What is self-rated health and why does it predict mortality? Towards a unified conceptual model. Social Science \& Medicine, 69(3), 307-316. doi: 10.1016/j.socscimed.2009.05.013.

Jylhä, M., Guralnik, J. M., Balfour, J., \& Fried, L. P. (2001). Walking difficulty, walking speed, and age as predictors of self-rated health: the women's health and aging study. The Journals of Gerontology Series A: Biological Sciences and Medical Sciences, 56(10), M609-M617.

Kautzky-Willer, A. (2014). Gendermedizin. Bundesgesundheitsblatt - Gesundheitsforschung - Gesundheitsschutz, 57(9), 1022-1030.

Knecht, M. K., Bauer, G. F., Gutzwiller, F., \& Hämmig, O. (2011). Persistent work-life conflict and health satisfaction - a representative longitudinal study in Switzerland. BMC Public Health, 11(1), 271. doi:10.1186/1471-2458-11-271.

Kroll, L. E., \& Ziese, T. (2009). Kompression oder Expansion der Morbidität? In: K. Böhm, C. Tesch-Römer \& T. Ziese (Hrsg.) Gesundheit und Krankheit im Alter [Beiträge zur Gesundheitsberichterstattung des Bundes] (S. 105-112). Berlin: Robert Koch-Institut.

Leinonen, R., Heikkinen, E., \& Jylhä, M. (2001). Predictors of decline in self-assessments of health among older people - a 5-year longitudinal study. Social Science \& Medicine, 52(9), 1329-1341. doi: 10.1016/ S0277-9536(00)00249-5.

Lynch, S. M. (2003). Cohort and life-course patterns in the relationship between education and health: A hierarchical approach. Demography, 40(2), 309-331.

McArdle, J. J. (2001). A latent difference score approach to longitudinal dynamic structural analysis. In: R. Cudeck, S. du Toit \& D. Sorbom (Hrsg.) Structural equation modeling: Present and future. A Festschrift in honor of Karl Jöreskog (S. 341-380). Chicago: Scientific Software International.

McArdle, J. J., \& Hamagami, F. (2001). Latent difference score structural models for linear dynamic analyses with incomplete longitudinal data. In: L. M. Collins \& A. G. Sayer (Hrsg.) New methods for the analysis of change (S. 139-175). Washington, DC: American Psychological Association.

Menning, S., \& Hoffmann, E. (2009). Funktionale Gesundheit und Pflegebedürftigkeit. In: K. Böhm, C. Tesch-Römer \& T. Ziese (Hrsg.) Gesundheit und Krankheit im Alter [Beiträge zur Gesundheitsberichterstattung des Bundes] (S. 62-78). Berlin: Robert Koch-Institut.

Nejati, V., Shirinbayan, P., Akbari Kamrani, A., Foroughan, M., Taheri, P., \& Sheikhvatan, M. (2008). Quality of life in elderly people in Kashan, Iran. Middle East Journal of Age and Ageing, 5(2), 21-25.

Pietschmann, P., Rauner, M., Sipos, W., \& Kerschan-Schindl, K. (2009). Osteoporosis: an age-related and gender-specific disease - a mini-review. Gerontology, 55(1), 3-12. 
Prentice, A. M. (2006). The emerging epidemic of obesity in developing countries. International Journal of Epidemiology, 35(1), 93-99. doi: 10.1093/ije/dyi272.

Schöllgen, I., Huxhold, O., \& Tesch-Römer, C. (2010). Socioeconomic status and health in the second half of life: findings from the German Ageing Survey. European Journal of Ageing, 7(1), 17-28.

Schöllgen, I., Morack, J., Infurna, F. J., Ram, N., \& Gerstorf, D. (2016). Health sensitivity: Age differences in the within-person coupling of individuals' physical health and well-being. Developmental Psychology, 52(11), 1944-1953. doi: 10.1037/dev0000171.

Sprangers, M. A., \& Schwartz, C. E. (1999). Integrating response shift into health-related quality of life research: a theoretical model. Social Science \& Medicine, 48(11), 1507-1515. doi: 10.1016/S02779536(99)00045-3.

Spuling, S. M., Wolff, J. K., \& Wurm, S. (2017a). Response shift in self-rated health after serious health events in old age. Social Science \& Medicine, 192, 85-93. doi: 10.1016/j.socscimed.2017.09.026.

Spuling, S. M., Wurm, S., Tesch-Römer, C., \& Huxhold, O. (2015). Changing predictors of self-rated health: Disentangling age and cohort effects. Psychology and Aging, 30(2), 462-474. doi: 10.1037/a0039111.

Spuling, S. M., Wurm, S., Wolff, J. K., \& Wünsche, J. (2017a). Heißt krank zu sein sich auch krank zu fühlen? Subjektive Gesundheit und ihr Zusammenhang mit anderen Gesundheitsdimensionen. In: K. Mahne, J. K. Wolff, J. Simonson \& C. Tesch-Römer (Hrsg.) Altern im Wandel. Zwei Jahrzehnte Deutscher Alterssurvey (DEAS) (S. 157-170). Wiesbaden: Springer VS.

Spuling, S. M., Ziegelmann, J. P., \& Wünsche, J. (2017b). Was tun wir für unsere Gesundheit? Gesundheitsverhalten in der zweiten Lebenshälfte. In: K. Mahne, J. K. Wolff, J. Simonson \& C. Tesch-Römer (Hrsg.) Altern im Wandel. Zwei Jahrzehnte Deutscher Alterssurvey (DEAS) (S. 139-156): Wiesbaden: Springer VS.

Trachte, F., Sperlich, S., \& Geyer, S. (2015). Kompression oder Expansion der Morbidität? Zeitschrift für Gerontologie und Geriatrie, 48(3), 255-262. doi: 10.1007/s00391-014-0644-7.

Wettstein, M., Schilling, O. K., \& Wahl, H.-W. (2016). "Still feeling healthy after all these years": The paradox of subjective stability versus objective decline in very old adults' health and functioning across five years. Psychology and Aging, 31(8), 815-830. doi: 10.1037/pag0000137.

World Health Organization (2002). Towards a common language for functioning, disability and health The International Classification of Functioning, Disability and Health. Genf: WHO.

Wills, T. A. (1992). The Role of Similarity in Coping Through Downward Comparison. In: L. Montada, S.-H. Filipp \& M. J. Lerner (Hrsg.) Life crises and experiences of loss in adulthood (S. 196-212). Hillsdale, NJ, US: Lawrence Erlbaum Associates.

Wolff, J. K., Nowossadeck, S., \& Spuling, S. M. (2017). Altern nachfolgende Kohorten gesünder? Selbstberichtete Erkrankungen und funktionale Gesundheit im Kohortenvergleich. In: K. Mahne, J. K. Wolff, J. Simonson \& C. Tesch-Römer (Hrsg.) Altern im Wandel. Zwei Jahrzehnte Deutscher Alterssurvey (DEAS) (S. 125-138). Wiesbaden: Springer VS.

Wurm, S., Lampert, T., \& Menning, S. (2009). Subjektive Gesundheit. In: K. Böhm, C. Tesch-Römer \& T. Ziese (Hrsg.) Gesundheit und Krankheit im Alter [Beiträge zur Gesundheitsberichterstattung des Bundes] (S. 79-91). Berlin: Robert Koch-Institut.

Zajacova, A., Huzurbazar, S., \& Todd, M. (2017). Gender and the structure of self-rated health across the adult life span. Social Science \& Medicine, 187, 58-66. doi: 10.1016/j.socscimed.2017.06.019. 
Open Access Dieses Kapitel wird unter der Creative Commons Namensnennung 4.0 International Lizenz (http:// creativecommons.org/licenses/by/4.0/deed.de) veröffentlicht, welche die Nutzung, Vervielfältigung, Bearbeitung, Verbreitung und Wiedergabe in jeglichem Medium und Format erlaubt, sofern Sie den/die ursprünglichen Autor(en) und die Quelle ordnungsgemäß nennen, einen Link zur Creative Commons Lizenz beifügen und angeben, ob Änderungen vorgenommen wurden.

Die in diesem Kapitel enthaltenen Bilder und sonstiges Drittmaterial unterliegen ebenfalls der genannten Creative Commons Lizenz, sofern sich aus der Abbildungslegende nichts anderes ergibt. Sofern das betreffende Material nicht unter der genannten Creative Commons Lizenz steht und die betreffende Handlung nicht nach gesetzlichen Vorschriften erlaubt ist, ist für die oben aufgeführten Weiterverwendungen des Materials die Einwilligung des jeweiligen Rechteinhabers einzuholen. 